\title{
Vertebral Artery Anomalies at the Craniovertebral Junction: A Case Report and Review of the Literature
}

\author{
Amir M. Abtahi ${ }^{1}$ Darrel S. Brodke ${ }^{1}$ Brandon D. Lawrence ${ }^{1}$ \\ ${ }^{1}$ Department of Orthopaedics, University of Utah, Salt Lake City, \\ Address for correspondence Brandon D. Lawrence, MD, Department \\ Utah, United States \\ of Orthopaedics, University of Utah, 590 Wakara Way, Salt Lake City, \\ UT 84108, United States (e-mail: brandon.lawrence@hsc.utah.edu).
} Evid Based Spine Care J 2014;5:121-125.

\begin{abstract}
Study Design Case report.

Objective The objective of this study was to report a case of an unstable C1 burst fracture in the setting of a vertebral artery anomaly at the craniovertebral junction.

Methods A 55-year-old man was admitted to the hospital with severe neck pain after falling approximately 15 feet and landing on his head. Computed tomography scan of the cervical spine revealed an unstable fracture of the $\mathrm{C} 1$ ring with magnetic resonance imaging evidence of a transverse ligament rupture as well as a congenital synchondrosis of the posterior arch of $\mathrm{C} 1$. He was neurologically intact. CT angiography (CTA) of the neck revealed an anomalous course of the right vertebral artery at the C1-C2 level.

Results Surgical intervention consisted of occiput-C3 fusion, thus avoiding the placement of $\mathrm{C} 1$ lateral mass screws and risking vertebral artery injury.

Conclusion We present a case of an unstable $\mathrm{C} 1$ burst fracture with an anomalous course of the right vertebral artery demonstrated by CTA. The presence of vertebral artery anomalies at the craniovertebral junction may prevent safe placement of C1

Keywords

- vertebral artery

- C1 synchondrosis

- C1 burst fracture lateral mass screws and therefore influence the treatment options for upper cervical spine pathologies. To minimize the risk of vertebral artery injury, we elected to perform an occiput to $\mathrm{C} 3$ fusion. Thorough assessment of the vascular anatomy is recommended before operative intervention in the upper cervical spine to minimize the risk of complications.
\end{abstract}

\section{Introduction}

Variations in the course of the vertebral artery have been previously described in the literature and tend to follow predictable patterns of variation. This is particularly true of lower cervical vertebral artery anatomy as it relates to the transverse foramina of C6 and C7. Less commonly known and described in the spine literature are the variations in vertebral artery anatomy in the upper cervical spine around the $\mathrm{C} 1$ lateral mass. The presence of these variations may influence treatment options for upper cervical spine pathology and may

received

December 20, 2013

accepted

June 2, 2014

in fact prevent commonly performed stabilization procedures. A case is presented that illustrates this vertebral artery anatomic variation at the craniovertebral junction and upper cervical spine, and the literature related to this subject is reviewed.

\section{Case Report}

A 55-year-old man presented to our institution with severe neck pain after falling 15 feet from a ladder and landing on his head. He was awake and alert at the time of presentation, had

(c) 2014 Georg Thieme Verlag KG Stuttgart · New York
DOI http://dx.doi.org/ 10.1055/s-0034-1386751. ISSN 1663-7976. 


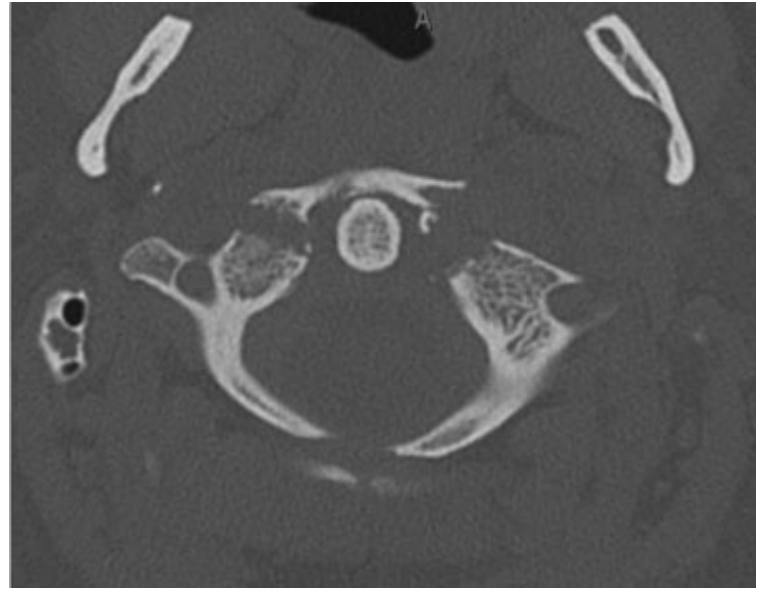

Fig. 1 Axial computed tomography demonstrating unstable $C 1$ burst fracture with an unfused posterior C1 synchondrosis.

no neurologic symptoms, and had a normal neurologic exam. A computed tomography scan of the cervical spine revealed an unstable fracture of the anterior ring of $\mathrm{C} 1$ and a synchondrosis of the posterior arch of C1 (-Fig. 1). Magnetic resonance imaging scanning demonstrated a transverse ligament rupture. CT angiography revealed a persistent first intersegmental artery (FIA) at the C1-C2 level with the FIA passing directly posterior to the caudal $\mathrm{C} 1$ lateral mass, thus preventing safe placement of a $\mathrm{C} 1$ lateral mass screw ( - Fig. 2A, B). To minimize the risk of vertebral artery injury, we elected to perform an occiput to $\mathrm{C} 3$ fusion.

A classic midline posterior cervical approach was used exposing the occiput cranially to the lateral masses of C3 caudally. We were able to identify the congenital defect in the posterior ring of $\mathrm{C} 1$. We began by placing an occipital plate on the posterior occiput. We then placed $\mathrm{C} 2$ pars screws bilaterally, followed by placement of bilateral C3 lateral mass screws and contoured rods. Fluoroscopy was used to confirm satisfactory placement of hardware and to guide fracture reduction before final fixation.
After all hardware had been placed, we then completely decorticated the inferior aspect of the skull, the posterior ring of $\mathrm{C} 1$, and the lamina and spinous processes of $\mathrm{C} 2$ and $\mathrm{C} 3$. We used tricortical iliac crest allograft secured with \#5 FiberWire (Arthrex, Naples, Florida, United States) as well as local autograft mixed with crushed cancellous allograft and DBX (Synthes, West Chester, Pennsylvania, United States) to facilitate fusion (-Fig. 3A, B).

The patient was neurologically intact postoperatively and mobilized well. He was pleased with his overall alignment and was discharged to home on postoperative day 5 in good condition. His 6-month postoperative evaluation revealed a well-healed fusion and the patient has returned to work.

\section{Discussion}

The presence of vertebral artery variation at the craniovertebral junction may influence treatment options for several upper cervical spine pathologies and should be identified preoperatively. This allows the practitioner to plan surgical intervention so as to minimize risk to the vertebral arteries. Variations in the course of the vertebral artery at the craniovertebral junction tend to follow predictable patterns. The anatomy of the vertebral artery and its variants can be most easily understood in the context of their embryonic development. The vertebral arteries develop from longitudinal anastomoses, linking the embryonic first to seventh cervical intersegmental arteries. During the course of normal development, the first to sixth cervical intersegmental arteries regress. The seventh cervical intersegmental artery persists and becomes the subclavian artery from which the vertebral arteries originate. $^{1}$

Three types of vertebral artery variation at the craniovertebral junction have been described ( $\mathbf{- F i g . 4 A - C})^{2,3}$ The most common variant is a persistent FIA, which arises when part of the embryonic FIA persists causing the vertebral artery to take an "anomalous" course and enter the spinal canal between $\mathrm{C} 1$ and $\mathrm{C} 2$ with absence of the normal vertebral artery branch. A recent magnetic resonance angiography
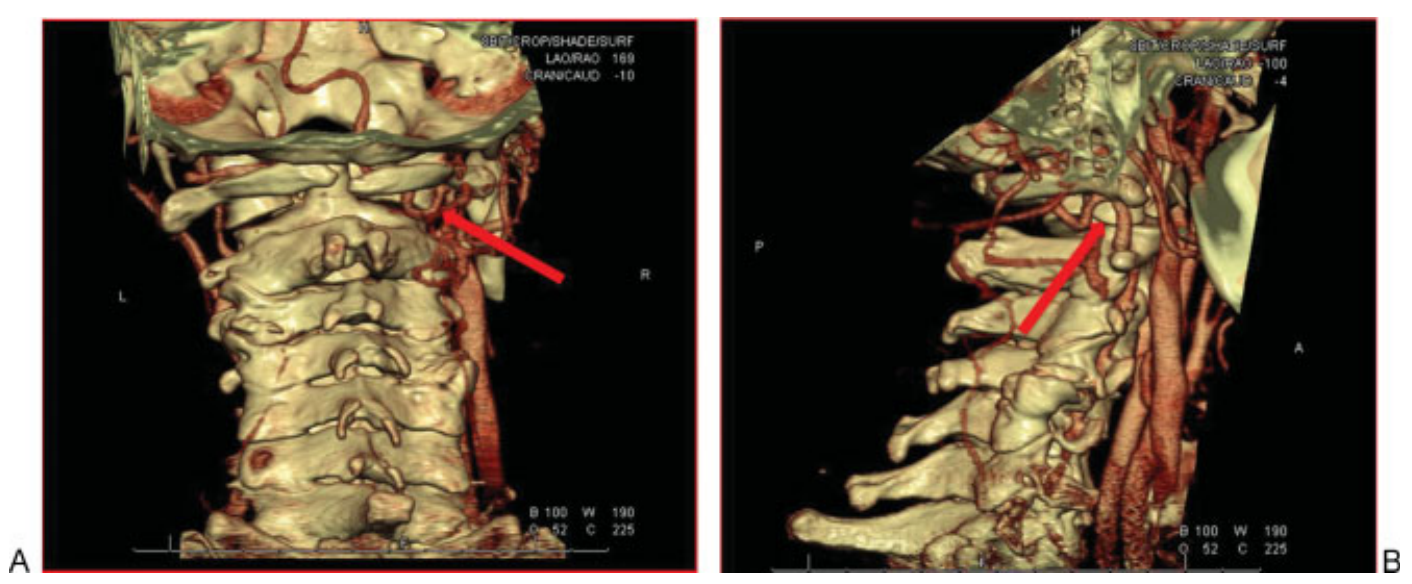

Fig. 2 (A, B) Three-dimensional CT angiography reconstructions demonstrating a persistent right side dominant first intersegmental artery entering the spinal canal between C1 and C2 (denoted by red arrow). The normal vertebral artery branch is absent. 


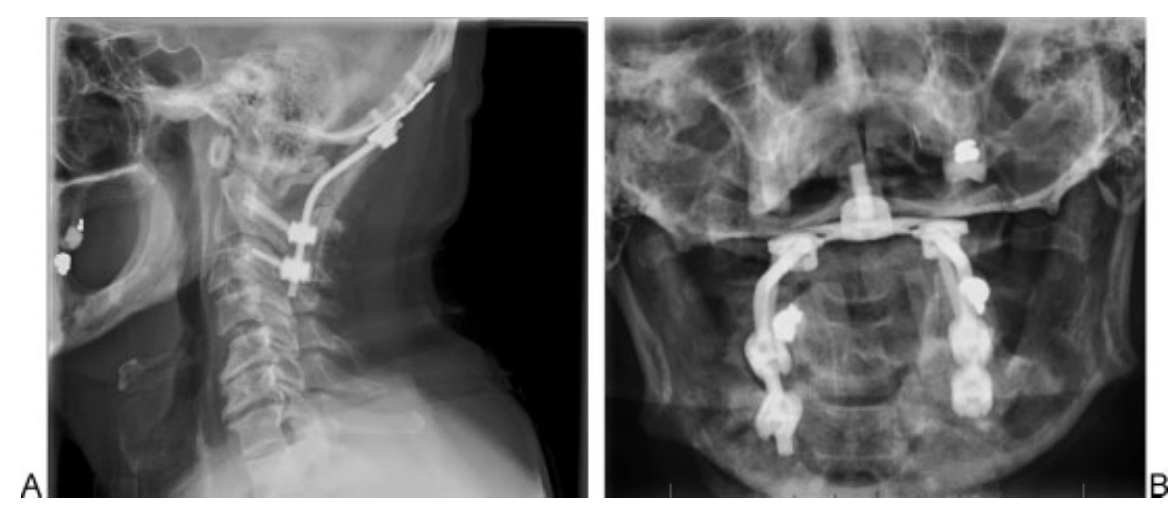

Fig. 3 (A, B) Anteroposterior open mouth and lateral postoperative radiographs showing occiput-C3 fusion construct.

(MRA)-based study has shown this variant to be present in $3.2 \%$ of patients. ${ }^{3}$ The next most common variant is an extracranial $\mathrm{C} 1 / 2$ origin of the posterior inferior cerebellar artery (PICA), present in $1.1 \%$ of patients. In this variant, the FIA continues to the PICA without reuniting with the vertebral artery. The third and least common variant is fenestration of the vertebral artery, present in $0.9 \%$ of patients. This variant occurs when there is both a normal vertebral artery branch as well as a persistent FIA. These branches then reunite within the spinal canal. All three of these variants may affect screw placement as the anomalous vertebral artery passes directly dorsal to the $\mathrm{C} 1$ lateral mass. Other less common variants have been described but are beyond the scope of this article.

Uchino et al reviewed MRAs from 2,739 patients and reported that the overall prevalence of vertebral artery anomalies at the craniovertebral junction is $5.0 \%$, and that in approximately $11 \%$ of these patients, the vertebral artery anomalies were bilateral. ${ }^{3}$ Yamazaki et al have reported an
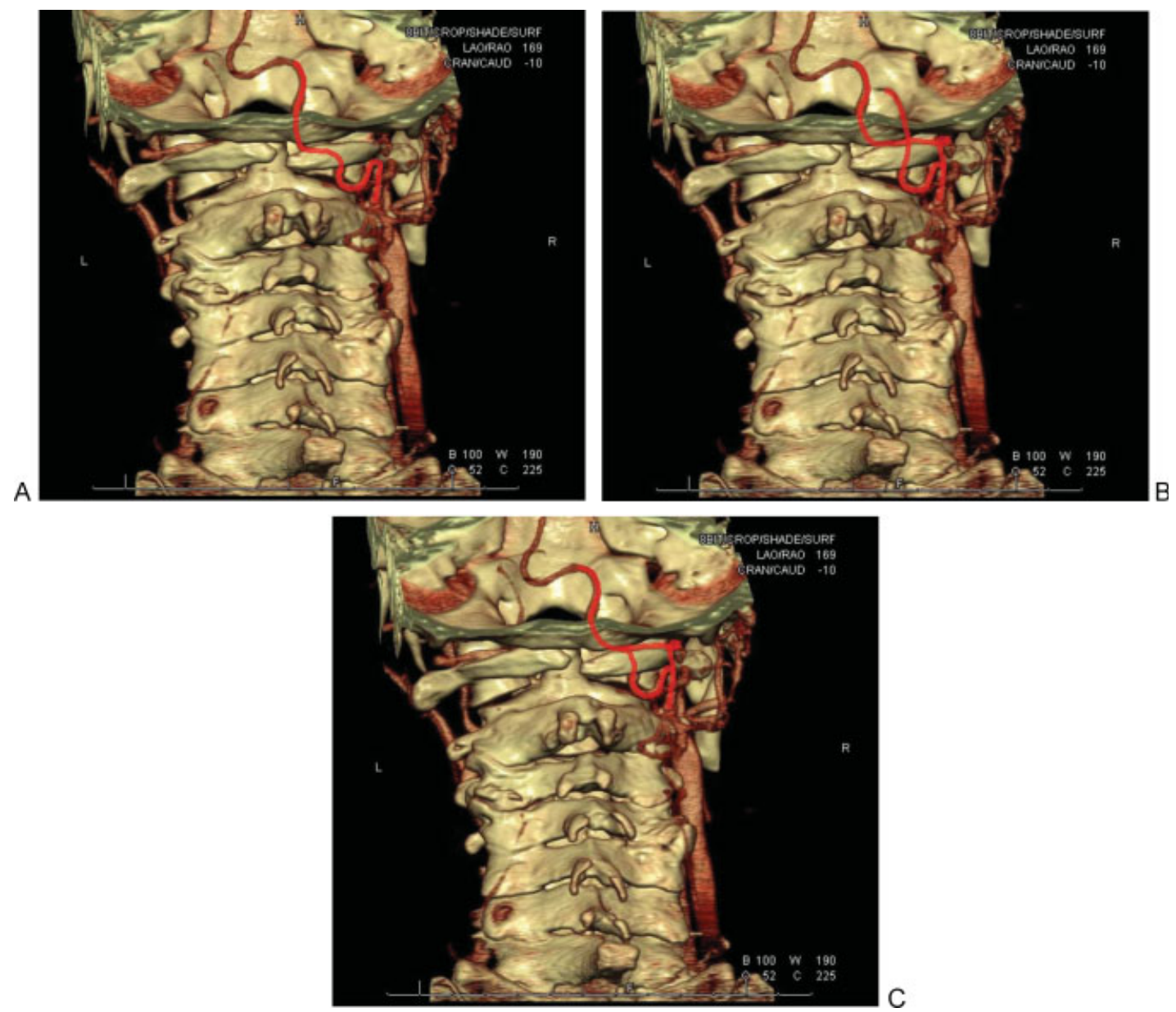

Fig. 4 (A-C) Illustration of persistent first intersegmental artery, extracranial C1/2 origin of the posterior inferior cerebellar artery, and fenestration of the vertebral artery. 
increased frequency of vertebral artery anomalies at the craniovertebral junction in patients with osseous anomalies at the craniovertebral junction (as was seen in this patient who had a posterior $\mathrm{C} 1$ synchondrosis) as well as in patients with Down syndrome. ${ }^{4,5}$

As demonstrated by the case presented above, because the persistent FIA enters the spinal canal between $\mathrm{C} 1$ and $\mathrm{C} 2$, it directly affects the ability to safely place $\mathrm{C} 1$ lateral mass screws. Attempted placement of $\mathrm{C} 1$ lateral mass screws may lead to vertebral artery injury. If these variations are identified preoperatively, surgical intervention may be planned so as to minimize the risk of a catastrophic vertebral artery injury. There are several reports in the literature describing alternative options for instrumentation in the presence of vertebral artery anomalies at the craniovertebral junction. Hong et al reported on the use of a superior C1 lateral mass entry point in a patient with left-sided FIA and traumatic cervical spine injury. ${ }^{6}$ This, of course, requires a complete absence of a vertebral artery remnant in the normal position superior to the $\mathrm{C} 1 \mathrm{ring}$. This technique, therefore, would only be possible in patients with a persistent FIA. Carmody et al reported on the use of $\mathrm{C} 1$ posterior arch screws in a patient with bilateral persistent FIAs and congenital fusion of $\mathrm{C} 2-\mathrm{C} 3$ in the setting of a type II odontoid fracture. ${ }^{7}$ Other options for fixation could include $\mathrm{C} 1-\mathrm{C} 2$ posterior wiring and $\mathrm{C} 1-\mathrm{C} 2$ transarticular screws, neither of which would have been possible in this case given the $\mathrm{C} 1$ synchondrosis and the unstable nature of the C1 burst fracture. These alternative methods have not been previously described in the literature.

In the case reported earlier, the patient was found to have a fracture of the anterior arch of $\mathrm{C} 1$ in association with a congenital cleft in the posterior arch. Although relatively well-defined criteria have been established for the management of traumatic $\mathrm{C} 1$ burst fractures in the absence of congenital anomalies of the $\mathrm{C} 1 \mathrm{ring}$, no criteria have been established for management of these injuries in the presence of congenital defects in the $\mathrm{C} 1$ ring. Attempted conservative treatment of unstable $\mathrm{C} 1$ burst fractures in the presence of congenital defects in the posterior ring of $\mathrm{C} 1$ has previously been reported to result in failure. ${ }^{8}$ In the case reported, $7.7 \mathrm{~mm}$ of displacement of the lateral masses of $\mathrm{C} 1$ relative to C2 was measured on the CT scan and evidence of transverse ligament injury was seen on MRI, therefore, meeting criteria for instability and operative treatment. Although standard operative treatment of an unstable $\mathrm{C} 1$ burst fracture would typically consist of $\mathrm{C} 1-\mathrm{C} 2$ fusion, we elected to perform an occiput to $\mathrm{C} 3$ fusion, eliminating the need to instrument the C1 lateral masses. It should be emphasized that before surgery, we had a detailed discussion with the patient re- garding the pros and cons of available surgical options. After discussing the risks and benefits of each option, the patient and surgical team decided that the safest and most reliable surgical option was occiput to $\mathrm{C} 3$ posterior fusion. The main disadvantage of this option is the loss of motion at the atlantooccipital joint. Another option, though not performed here, would be to instrument the occiput-C1 level without fusion. If this option was chosen, occiput-C1 instrumentation could be subsequently removed after $\mathrm{C} 1-\mathrm{C} 3$ arthrodesis occurred, potentially allowing the return of motion at the atlantooccipital joint. Ultimately, the method of instrumentation should depend on the surgeon's experience and comfort with available techniques.

\section{Disclosures}

Amir M. Abtahi, none

Darrel S. Brodke, Consultancy: Amedica, DePuy Synthes; Royalties: Amedica, DePuy, Medtronic

Brandon D. Lawrence, none

\section{References}

1 Padget DH. The development of cranial arteries in the human embryo. Contrib Embryol 1948;32:207-261

2 Tokuda K, Miyasaka K, Abe $\mathrm{H}$, et al. Anomalous atlantoaxial portions of vertebral and posterior inferior cerebellar arteries. Neuroradiology 1985;27(5):410-413

3 Uchino A, Saito N, Watadani T, et al. Vertebral artery variations at the C1-2 level diagnosed by magnetic resonance angiography. Neuroradiology 2012;54(1):19-23

4 Yamazaki M, Koda M, Aramomi MA, Hashimoto M, Masaki Y, Okawa A. Anomalous vertebral artery at the extraosseous and intraosseous regions of the craniovertebral junction: analysis by three-dimensional computed tomography angiography. Spine (Phila Pa 1976) 2005;30(21):2452-2457

5 Yamazaki M, Okawa A, Hashimoto M, Aiba A, Someya Y, Koda M. Abnormal course of the vertebral artery at the craniovertebral junction in patients with Down syndrome visualized by threedimensional CT angiography. Neuroradiology 2008;50(6): 485-490

6 Hong JT, Jang WY, Kim IS, et al. Posterior C1 stabilization using superior lateral mass as an entry point in a case with vertebral artery anomaly: technical case report. Neurosurgery 2011; 68(1, Suppl Operative ):246-249, discussion 249

7 Carmody MA, Martin MD, Wolfla CE. Persistent first intersegmental vertebral artery in association with type II odontoid fracture: surgical treatment utilizing a novel $\mathrm{C} 1$ posterior arch screw: case report. Neurosurgery 2010;67(1):210-211, discussion 211

8 Hudek R, Wanner G, Simmen HP, Werner CM. C1 fracture in a patient with a congenital cleft in the posterior arch: report on a failed conservative treatment. BMJ Case Rep 2013;2013 


\section{Editorial Perspective}

Awareness of anatomic variations and picking up on them before engaging in spine surgery is desirable. Discerning anatomic and radiographic subtleties in the spine is a sometimes humbling, lifelong learning opportunity and not always straightforward. The incidence of vertebral artery malformations and norm variants is a case in example and a good application for case reports, as their occurrence is usually sporadic for most practitioners. The calculation of an actual incidence of a norm variant, however, is a different story. The quoted incidence of 0.1 to almost $2 \%$ reflects the relative statistical uncertainty in trying to calculate occurrence rates from existing databases. For instance, the above quoted Uchido study relied on an MRI library of spine patients in Japan, introducing ethnicity and underlying spine pathology as potential sources of bias. The actual finding of a vertebral artery anomaly will remain an incidental occurrence for most practitioners, but by sharpening our awareness, hopefully we can consistently help increase the safety of spine surgery. This article by Dr. Lawrence with its very clear figures and the helpful comments by Dr. Molinari do their part in sharpening our senses toward looking for vertebral anomalies in our routine studies as part of our routine preoperative planning process. 